\title{
Nachbehandlung zur Lebensdauerverlängerung
}

Durch eine gezielte Nachbehandlung der Schweißnähte, vor allem der Schweißnahtübergänge, kann bewirkt werden, dass die Betriebsfestigkeit und Lebensdauer dynamisch belasteter geschweißter Metallkonstruktionen erheblich gesteigert werden. Das wird erreicht durch Schleifen, Strahlen, Hämmern oder durch eine Wärmebehandlung. Durch diese Einwirkung auf die Schweißnahtübergänge wird insbesondere eine Reduzierung von Kerbwirkungen erreicht.

Auch eine Verfestigung der Randschicht und das Einbringen von Druckeigenspannungen führen zu einer Erhöhung des Widerstandes gegen Rissbildung und -ausbreitung durch Überlagerung der Kerbspannungen mit Druckeigenspannungen. Nach solchen Behandlungen können die Schweißverbindungen noch durch eine Beschichtung mit einem korrosionsbeständigen Werkstoff als passivem Korrosionsschutz weiter geschützt werden. 